Aus der chirurgischen Universitätsklinik Rostock (Direktor: Geh. Rat Prof. Dr. W. Müller).

\title{
Zur Klinik, pathologischen Anatomie und Ätiologie des Angioma arteriale racemosum, besonders der Extremităten.
}

Von Dr. Egbert Schwarz, I. Assistenten der Klinik.

Die häufigste Lokalisation des sogenannten Angioma arteriale racemosum ist der Kopf, besonders im Bereich der Schädeldecken. Aber auch im Inneren der Schädelhöhle sind ähnliche Bildungen unter diesem oder anderen Namen beschrieben worden (Emanuel (9), Steinheil (29), Kalischer (17), Deetz (6), Drysdales (7), Simmonds (28)). Demgegenüber tritt die Lokalisation solcher "Neubildungen" an den Extremitäten stark zurück. Immerhin verfügt die Literatur doch über eine ganze Reihe derartiger Beobachtungen. Wagner (32) veröffentlichte 1894 einen Fall am Finger und stellte 15 andere im Bereich der oberen Extremität aus der bis dahin bekannten Literatur zusammen. Diese 15 Fälle sind den bekannten Arbeiten von H e in e (I 5) und S ch ü ck (25) entnommen. Drei andere scheinen sich an der unteren Extremität befunden zu haben. Dazu kommen aus dem Jahre 1897 noch drei Beobachtungen von Engelbrecht (IO), eine weitere von Borchardt (3) aus dem Jahre 1904, ein Fall von Rankenangiom an der unteren Extremität von Froehlich (I2) aus dem Jahre 1902 und schließlich eine Beobachtung von Aneurysma cirsoides des Armes und Vorderarmes von $\mathrm{Gabryszewski}$ (14). In der Arbeit von Fröhlich findet sich auch eine Übersicht der übrigen an der unteren Extremität beobachteten Rankenangiome; es sind im ganzen neun (v. Si e. bold (26), Breschet (4), Baum (2), Wilms (33), Panas 22), Edmunds (8), Siegmund (27), Israel (I6), Nicoladoni (21). Eine Statistik, die größere Zahlen über racemöse Angiome an der oberen Exitremität aufweist, stammt von Mink ( 32 Fälle), 
von denen 28 Beobachtungen einer Zusammenstellung von $\mathrm{Po}_{\mathrm{o}}$ laillon entnommen sind, doch sind nach Wagner (l.c.) von diesen nur I 3 Fälle als echte arterielle Rankenangiome anzusehen. Die übrigen seien mit andersartigen Gefäßerkrankungen, wahren Aneurysmen, Aneurysmata arteriovenosa, Angioma simplex und diffusen Phlebarteriektasien verwechselt worden.

Nun hatte ich kürzlich Gelegenheit, die Beobachtung einer Gefäßerkrankung zu machen, die wohl in das bezeichnete Gebiet der sogenannten Rankenangiome hineingehören mag. Die Beobachtung war kurz folgende:

Vor einigen Wochen wurde in die Klinik ein $45 \mathrm{j}$. Mann aufgenommen. Er gab an, als Kind immer gesund gewesen zu sein. In den Jahren 1900-r 903 sei er in China gewesen und habe am dortigen Feldzuge teilgenommen. In der Zeit hätte er Ruhr und Malaria gehabt. Seit 1905 leide er an rheumatischen Beschwerden, besonders der Hände. Während des großen Krieges sei er Soldat gewesen, habe jeden Dienst gut machen können. In den Beinen hätte er nie Beschwerden gehabt.

IgI9 wurde er wegen eines Pleuraempyems, das sich an nach Grippe entstandene Lungenentzündung angeschlossen hatte, operiert. Mai 1920 sei er an einer Nierenentzündung erkrankt, in deren Verlauf krampfartige Zustände mit Bewußtseinsstörungen aufgetreten seien, so daB er eine Zeitlang in die Nervenklinik hätte verlegt werden müssen.

Anfang Nov. I920 hätte er plötzlich eines Tages ein stechendes Gefühl im 1. Oberschenkel bekommen. Die Schmerzen im 1. Bein, die nun immer häufiger wurden und sich beim $\mathrm{Herunterhängenlassen} \mathrm{des}$ Beines verstärkten, wurden so unerträglich, daß er das Bett hüten mußte. Als er Ende Nov. einen Versuch machte, aufzustehen, bekam er plötzlich sehr heftige Schmerzen an der gleichen Stelle des 1. Oberschenkels und bemerkte seitdem eine immer mehr zunehmende Schwellung an der Vorderseite des Oberschenkels.

Anfang Dez. kam er in die Klinik und machte einen außerordentlich blassen, anämischen Eindruck. Außer einer ziemlich erheblichen Bronchitis war an den inneren Organen nichts Besonderes nachzuweisen.

Der 1. Oberschenkel war in seiner oberen Hälfte geschwollen. Die größte Schwellung bezog sich auf die Vorderfläche der oberen Hälfte des Oberschenkels, reichte nach oben bis zum Leistenbande, nach unten bis zur Mitte des Oberschenkels, nach innen bis in die Adduktorengegend und nach außen bis zur äußeren Peripherie des Oberschenkels. Die Geschwulst war von halbkugeliger Gestalt, glatter Oberfläche, im unteren 'Teil ziemlich druckempfindlich und fluk- 
tuierend. Temp. und Puls waren normal. Weder auskultatorisch noch palpatorisch fanden sich Schwirren oder Geräusche im Bereich der Schwellung, geschweige denn Pulsation. Wassermannsche Reaktion im Blut negativ. Im Urin Spuren von EiweiB, im Sediment einige Leukocyten, keine Zylinder.

Es wurde an einen Tumor oder Absze $B$ am Oberschenkel gedacht, erst im Beginn der Operation an ein Aneurysma.

Bei der Operation wurde folgender Befund erhoben: Schnitt etwa der Außenkante des Sartorius entsprechend. Auseinanderziehen der Wundränder, Wegziehen des Sartorius nach der medialen Seite hin und Spaltung der tieferen Fascienblätter. Dabei kommt man plötzlich in eine doppelt-faustgroße Höhle, aus der sich unter Spannung stehende Blutgerinnselmassen entleeren. Bei dem Versuch, sie vollkommen auszuräumen, entsteht eine stärkere frische Blutung, so daß die Höhle schnell tamponiert werden muß, da die Beherrschung der Blutung von hier aus in dem unübersichtlichen Gewebe unmöglich erscheint. Es wird deshalb ein zweiter, mehr medialer Schnitt zur Freilegung der A. femoralis angelegt, die Arterie selbst freipräpariert und angeschlungen. Dann wird die Abgangsstelle der A. profunda femoris aufgesucht. Es zeigt sich, da $B$ sie fast ebenso stark ist wie die A. femoralis. Die Profunda femoris wird weiter nach abwärts verfolgt, wobei die Muskeln des Oberschenkels weit auseinander gezogen werden können. Dabei erweist sich, daß die Arterie sich bald in zahlreiche Äste aufteilt, die, ebenso wie der Hauptstamm, eine ungewöhnliche Stärke haben, so daß ein größerer Teil der Verzweigungen dem Lumen der A. ulnaris oder radialis entspricht. Alle Äste pulsieren stark, sind auffallend geschlängelt und ziehen, ohne sich wesentlich zu verengern, in die einzelnen Muskeln hinein. Der schon früher öfters gezogene Vergleich mit Regenwürmern trifft auch für das vorliegende Bild zu. Im unteren Abschnitt des in geschildeter Weise veränderten Gefäßbaumes der A. profunda kommt man in die zuerst freigelegte, mit Blutgerinnseln gefüllte Höhle. Die A. profunda wird, da eine Blutung aus einem ihrer Äste wahrscheinlich ist, kurz nach dem Abgang aus der Femoralis unterbunden und ein mehrere Zentimeter langer Abschnitt ihres Stammes reseziert.

Durch die Unterbindung hört das Pulsieren der Arterienäste jedoch nicht vollkommen auf, wenngleich die Pulsation wesentlich vermindert wird. Auch die Blutung aus der großen Aneurysmahöhle hat nun soweit nachgelassen, $\mathrm{da} B$ an eine nähere Besichtigung derselben herangegangen werden kann. Nach Ausräumung der Blutgerinnsel finden sich in der Tiefe wiederum zahlreiche geschlängelte Arterienäste. Man hat durchaus den Eindruck, als ob die Wundhöhle von einem Netz dieser geschlängelten Gefäße umgeben sei. Es blưtet nun aber noch von der medialen Seite der Höhle her, so da $B$ als Ursprungsort der Blutung ein Ast des Verzweigungsgebietes der A. obturatoria angenommen werden muß. Das Gebiet der Adduktoren 
wird freigelegt. Auch hier finden sich überall er we it er te und ge schlängelte Arterienäste. Die Blutung kommt aus einem der gröBeren Äste der A. obturatoria. Die blutende Stelle ist z. T. noch mit Blutkoageln bedeckt. Es werden eine Reihe der größeren Arterienäste gefaßt und unterbunden, sowie größere Abschnitte der geschlängelten Arterien sowohl aus dem Gebiet der Obturatoria als auch der Profunda femoris mit der Umgebung exstirpiert.

Bei genauerer Betrachtung fallen an manchen Stellen kleine, sackartige Erweiterungen einzelner Arterienäste auf, von denen die größte an der Stelle der Blutung aus dem Ast der A. obturatoria gelegen ist. Ihre Wand ist nun allerdings nicht mehr ganz vollständig, das Aneurysma ist geplatzt und scheint die große Blutung zwischen die Muskeln verursacht $z u$ haben. Tamponade der Wundhöhle, Hautnähte. Weiterer Verlauf ohne Störung nach anfänglicher eitriger $\mathrm{Ab}$ sonderung aus den Tamponstellen.

Nach diesem makroskopischen Befund handelt es sich also bei einem durch längere Krankheit stark geschwächten und sehr anämischen Mann um eine Gefäßerkrankung im Gebiet der A. profunda femor. und obturatoria, deren Hauptmerkmale makroskopisch eine Verdickung und Erweiterung der beiden Arterien mit all ihren Ästen, eine außergewöhnliche Schlängelung derselben, sowie sackartige aneurysmatische Erweiterungen an einzelnen Stellen sind. Das eine dieser Aneurysmen ist ohne eine nachweisbare Ursache geplatzt und hat die große Blutung unter Entwickelung des Tumors am Oberschenkel hervorgerufen.

Die mikroskopischen Präparate, welche anzusehen Herr Professor Dr. $\mathrm{H}$ u e $\mathrm{ck}$ die große Liebenswürdigkeit hatte und über deren Befund er mir seine Ansicht darlegte, wofür ich ihm auch an dieser Stelle meinen besten Dank aussprechen darf, boten folgendes Bild:

Der Hauptstamm der A. profunda femoris zeigt im wesentlichen den normalen Aufbau einer Arterie mittleren Kalibers. Die Intima ist dün, an 2 Stellen sieht man in ihr 2 kleine Degenerationsherde, die mit homogener Masse angefüllt sind und sich mit Hämatoxylin blau gefärbt haben. Offenbar handelt es sich um kleine Kalkherde. Die Membrana elastica interna ist überall entweder als dicker, gewellter Streifen, oder aufgefasert in mehrere Lamellen, zu erkennen. Die Media ist breit und besteht aus glatten Muskelfasern, die durch verhältnismäßig breite Bindegewebszüge voneinander getrennt sind. Sowohl in der Intima als auch der Media haben sich elastische Fasern gut färben lassen, die hier aber eine unregelmäßige Verteilung zeigen. Die Adventitia hat normalen $\mathrm{Bau}$, ist nicht verdickt und enthält viel elastische Fasern. 
In anderen Schnitten sieht man in jedem Gesichtsfeld eine ganze Reihe von Gefäßquerschnitten, von denen die größten etwa dem Lumen der A. radialis gleich kommen. Die Gefäße liegen in einem chronischen Granulationsgewebe, zum Teil in derbes Narbengewebe eingebettet. In diesem sieht man zahlreiche kleinere Gefäße von muskulärem Typus und zu Gruppen angeordnete Kapillaren, die von kleinen Rundzellenanhäufungen umgeben sind. Außer zahlreichen Hämorrhagien und stellenweisen Anhäufungen von Blutpigment findet sich hier nichts besonders Charakteristisches.

Die Gefäße selbst lassen Intima, Media und Adventitia gut erkennen. Diese ist überall reichlich mit gekräuselten elastischen Fasern versehen und geht direkt in das narbige, die Gefäße umgebende Gewebe über. Die Media erscheint an manchen Gefäßen verdickt und besteht aus glatten Muskelfasern und Bindegewebe. Hie und $\mathrm{da}$ sind auch dünne, elastische Fasern nachweisbar. Aber nicht überall ist der Aufbau der Media ein so gleichmäBiger. Denn es finden sich einerseits, meist an der Grenze zur Adventitia, streifen. förmig in ihr angeordnete Rundzelleninfiltrate; anderseits aber auch narbige Prozesse in Form von die Wand des Gefäßes quer durchsetzenden, ziemlich kernarmen Bindegewebszügen, so da $B$ man hier narbig ausgeheilte Rupturstellen der Gefäßwand anzunehmen geneigt ist, zumal in der Umgebung der Stellen die Residuen alter Hämorrhagien nachzuweisen sind. Unregelmäßig verteilt findet man in der Media mancher Gefäße kleine Degenerationsherde, die meist gegen die Grenze zur Intima hin lokalisiert sind. Die Membrana elastica interna ist überall gut ausgebildet, nur an einzelnen Stellen liegen kleine Degenerationsherde gerade in ihrem Verlauf und scheint sie stellenweise zu beiden Seiten solcher Herde zu fehlen.

Die stärksten Veränderungen zeigt die Intima. An vielen $\mathrm{Ge}$ fäßen ist sie abschnittweise verdickt und springt in Form kleiner Protuberanzen oder halbmondförmiger Verdickungen in das Innere des Gefäßes vor. $\mathrm{Da} B$ es sich tatsächlich um Intimawucherungen hanhelt, beweist der verhältnismäßig große Reichtum an elastischen Fasern; an einigen Gefäßen ist die Intimawucherung so stark, daß das Gefäßlumen vollkommen durch sie verschlossen ist.

Andere, meist größere Gefäße zeigen geringere Veränderungen an der Intima; ihr Lumen ist aber an vielen Stellen ganz oder fast ganz durch organisierte und vaskularisierte Thromben verschlossen. Viele der die Thromben durchziehenden Gefäße sind prall mit roten Blutkörperchen gefüllt. Einzelne Teile der Thromben weisen kalkige Degenerationsherde auf. Kombinationen zwischen Intimawucherungen und Thrombenbildung sind häufig zu sehen.

Die Wand des Aneurysmas zeigt ausgesprochene Schichtung und imponiert zunächst als dilatierte Gefäßwand, was aber nicht zutrifft, da sie lediglich aus geschichtetem Bindegewebe ohne elastische 
Fasern aufgebaut ist. In dem gleichen Präparat ist an einem Rande der halbe Querschnitt eines großen Gefäßes zu sehen, das in seinem ganzen Umfang etwa dem Stamme der oben beschriebenen A. profunda femoris entsprechen würde, sonst aber keine Veränderungen zeigt.

Was nun endlich das Präparat anbetrifft, das aus dem Gebiet der A. obturatoria gewonnen wurde und da $B$ Gefäß enthält, aus dem die Blutung erfolgte, so sieht man im Querschnitt an einer Seite des Gefäßes deutliche Intimawucherungen, andererseits eine starke Durch. setzung der Media mit Bindegewebe. Die Intima ist durch Blutungen von der Media abgehoben. Wo die Blutungen am zahlreichsten werden, findet sich die Rupturstelle des Gefäßes, deren Ränder zerfetzt sind. Auch das umliegende Gewebe ist von Blutungen und Rundzellen durchsetzt.

Fettige Degeneration in den Gefäßwänden fand sich nirgends, wie sie z. B. von $\mathrm{K} \ddot{\mathrm{u}} \mathrm{mmell}$ in einem Fall beschrieben wurde. Alle Gefäße zeigen die Characteristica der Arterien, dicke Wandung, dicke Membrana elastica interna. Venen fanden sich nur in geringer Zahl.

Wenn ich kurz zusammenfasse, so bestehen die histologischen Merkmale im wesentlichen in degenerativen Veränderungen der Arterienwände, d. h. bindegewebig-narbigen Prozessen der ganzen Wanddicke, Kalkherdchen der Intima und Media, starken Intimawucherungen, die halbmondförmig in das Lumen der Gefäßrohre hineinragen oder dieselben vollkommen verschließen; ausgedehnten Thromben verschiedenen Alters, die gleichfalls zum teilweisen oder vollkommenen Verschluß der Gefäße geführt haben.

Bei der Behandlung war im vorliegenden Falle nur ein Weg möglich, nämlich der der Unterbindung der zuführenden Arterienstämme, an die die Ausräumung der Blutgerinsel und Exzision und doppelte Unterbindung einer ganzen Reihe kleinerer Arterienverzweigungen angeschlossen werden konnte. Auf eine nähere Besprechung der Behandlung des Rankenangioms möchte ich nicht eingehen, weil das bereits ausführlich in zahlreichen Arbeiten geschehen ist (Heine, $\mathrm{Krause}, \mathrm{Körte}, \mathrm{Clair}$ mont, Lieblein, Siegmund, Heineke, Heidenhain, Ali Krogius, Kümmell, Wagner, Engelbrecht u. a.). Genaue Literaturangaben in den einschlägigen Fragen finden sich übrigens hauptsächlich bei H e in e (15), K ö r t e (19), S c h ü c k (25), $\mathrm{Keppler}$ (18) und in der neuesten Arbeit von $\mathrm{O} . \mathrm{S} \mathrm{ch} \mathrm{m} \mathrm{it} \mathrm{t} \mathrm{(24).}$ 
Die Anregung, mich mit den Fragen der pathologischen Anatomie und der Ätiologia der in Frage stehenden Gefäßerkrankung näher zu befassen, wurde mir durch meine Beobachtung gegeben und ich möchte deshalb mit einigen Worten näher auf sie eingehen. Unter dem Namen des Angioma arteriale racemosum verstehen wir im allgemeinen eine mehr oder weniger begrenzte "Geschwulstbildung" im Gebiet eines Arterienstammes oder seiner Verzweigungen mit Hypertrophie, Dilatation und starker Schlängelung der die Neubildung zusammensetzenden Gefäße. Bald erstreckt sich der von der Erkrankung ergriffene Bezirk über eine größere Fläche, bald bildet sie eine mehr zirkumskripte Schwellung, z. B. an Hand oder Fingern. Dann wieder ergreift sie ein größeres Gebiet von Arterien oder sogar mehrerer Arterien, wie beispielsweise in den Fällen von Engelbrecht (l.c.), Gabryczewski (l. c.) u. a., bei denen die Erkrankung sich nicht nur auf die Arterien der Hand, sondern auch die Stämme der Radialis, Ulnaris und Interossea, ja der Brachialis erstreckte. Häufig ist die Beobachtung von zylindrischen oder rundlichen, sackartigen aneurysmatischen Erweiterungen der Arterienwand. Als Paradigma für das Rankenangiom kann der mehrfach ab. gebildete Fall von $\mathrm{Clairmont}$ gelten, als gutes Beispiel für die Lokalisation im Gehirn die beiden Fälle von Simmonds, bei dessen erster Beobachtung es sich um das Ergriffensein eines ganzen Arterienbaumes, im zweiten um die Erkrankung bloß eines Teiles eines Arterienstammes handelte.

Wie verschieden die makroskopischen Bilder des Rankenangioms sind, ersieht man schon aus diesen wenigen Hinweisen. Ebenso differieren naturgemäß auch die Definitionen, die für dasselbe geprägt wurden. Virchow (3I) betonte das Charakteristikum, daß das Rankenangiom zusammengesetzt sei ,aus stark verdickten, gleichmäßig oder sackartig erweiterten, verlängerten und daher rankenartig aufgerollten Gefäßen eines gröBeren Arterienbezirks". Er hat als erster das Rankenangiom in das Gebiet der Geschwülste eingereiht und es von den aneurysmatischen Bildungen abgrenzen zu müssen geglaubt. Er betonte die Entstehung des Rankenangioms aus einer Telangiektasie oder einem Trauma. He in e (1. c.) äußerte sich schon etwas vorsichtiger, indem er betonte, da $\beta$ es sich um eine „bald mehr, bald weniger 
zirkumskripte, mit dem $\mathrm{Ch}$ arakter einer Geschwulst auftretende Erweiterung eines bestimmten arteriellen Gefäßbezirks bis in seine kapillaren Endverzweigungen hinein" handelt. Er betont also weniger scharf die Neubildung, im Gegensatz zu W a g n e r, der das größte Gewicht auf die „Neubildung arterieller oder arterienähnlicher Gefäße von ausgesprochen progressivem Charakter, einhergehend mit exzentrischer Hypertrophie bereits bestehender Arterien kleineren Kalibers" legt.

Die Verschiedenheit der pathologisch-anatomischen Bilder, die unter dem gleichen oder fast gleichen Namen beschrieben sind, liegt klar auf der Hand. Mehr Übereinstimmung dagegen scheint in einem anderen Punkt $\mathrm{zu}$ herrschen und scheinen die meisten sich darüber einig zu sein, daß es sich um eine echte Neubildung von Gefäßen im Sinne der Geschwulst handelt. Erst in neuerer Zeit ist an dieser Auffassung bereits mehr oder weniger Kritik geübt worden. Ich komme darauf noch gleich zurück. Zuvor möchte ich aber noch kurz auf die histologischen Veränderungen hinweisen. Auch hier kann von einem einheitlichen Bild nicht gesprochen werden. Histologische Untersuchungen liegen leider lange nicht von allen Beobachtungen vor. Wer sich genauer über die bekannten histologischen Befunde orientieren möchte, findet in der neuesten Arbeit von O. Schmitt (1. c.) ein genaueres Referat. Bald sind die histologischen Bilder normalen Arterien gleich, bald findet sich Hyperplasie aller drei Gefäßschichten, bald bezieht sich diese aber nur auf eine oder die andere Schicht. Häufig wiederkehrend ist die Beschreibung von Intimawucherungen, die buckelförmig in das Gefäßlumen hineinragen, wie auch ich sie in meinem Fall nachweisen konnte. Ganz unregelmäßig sind die Befunde an Media und Adventitia. Entzündliche Veränderungen der Gefäßwände finden sich angeblich niemals, worauf auch $\mathrm{Simmonds}$ in seinen Fällen hinweist. Einige fanden degenerative Prozesse in der Media und Adventitia, bei anderen fehlten solche, doch wurde dann Verfettung oder Atrophie der Gefäßschichten beobachtet ( $\mathrm{K}$ ü m mell).

Diese kurzen Bemerkungen zur Histologie des Rankenangioms mögen gleichfalls genügen, um zu zeigen, wie wenig Einheitlichkeit auch hinsichtlich der mikroskopischen Veränderungen herrscht. Man wird deshalb wohl berechtigt sein, zu sagen, daß 
lange nicht alles, was unter dem Namen arterielles Rankenangiom beschrieben wurde, diesen Namen mit Recht trägt, sondern daß es sich vielleicht in klinischer Beziehung öfter um gleichartige, pathologisch-anatomisch aber ganz verschiedenartige Dinge handelt, die also klinisch zu diagnostischen Verwechselungen führen können, aber in ätiologischer Hinsicht möglicherweise nichts miteinander zu tun haben. $\mathrm{Zu}$ ähnlichen Gedanken kam ja bereits $\mathrm{W}$ a gne $\mathrm{r}$ bei seiner Bearbeitung der razemösen Angiome an der oberen Extremität und sonderte aus anderen Statistiken, wie ich bereits zu Anfang erwähnte, alles aus, was auf Grund seiner Anschauungen über das wahre arterielle Rankenangiom seiner Ansicht nach nicht dazu gehörte. Er ging von dem Grundsatz aus, $\mathrm{da} ß$ es sich beim wahren Rankenangiom immer um eine vollkommene Neubildung von Gefäßen, bzw. um „einen Übergang von bereits bestehenden Gefäßen niederer Ordnung zu solchen höherer Ordnung" handelt. Schon in dieser Definition liegt meines Erachtens eine gewisse Unklarheit; denn es ist doch zum mindesten zweifelhaft, ob man einen durch Wachstum bewerkstelligten Übergang von Gefäßen niederer Ordnung in solche höherer Ordnung als Neubildung im Sinne der Geschwulst ansehen kann; oder ob nicht vielmehr eine solche angebliche Überführung von Gefäßen niederer in solche höherer Ordnung auch durch andere krankhafte Prozesse in einem Gefäßbezirk möglich ist. Die tatsächliche anatomische Grundlage für die Annahme Wagners ist ja nur eine Hypertrophie der Gefäßwand, Schlängelung der Gefäße und Dilatation, sowie mehr oder weniger ausgeprägte degenerative Prozesse in der Gefäßwand. Lassen sich nicht analoge pathologisch-anatomische Veränderungen auch bei anderen Erkrankungen finden? Ich möchte z. B. an die Gefäßveränderungen bei der einen oder anderen Form der Arteriosklerose oder an die Hypertrophie und Dilatation des Herzens bei Arteriosklerose oder bei Endo- oder Myocarditis erinnern.

Immerhin mag es sich in einer Reihe von Beobachtungen, besonders denen, wo die Gefäßveränderung nicht diffus, sondern eine ganz umschriebene tumorähnliche ist, wirklich um neugebildete Gefäße handeln. Ob man diese Neubildungen aber tatsächlich als Geschwustbildungen anzusprechen berechtigt ist, möchte ich noch dahingestellt sein lassen. Was gegen eine wahre 
Geschwulst spricht, ist einerseits der meistens regelmäßige Bau der Bildungen, in denen insbesondere die Gefäßwände alle einen mehr oder wenifger typischen Aufbau erkennen lassen, ganz abgesehen davon, daß in den Arterienwänden degenerative Veränderungen verschiedener Art vorliegen. Eine einseitige Hypertrophie der einen oder anderen GefäBschicht kann man doch nicht als atypisches Wachstum im Sinne einer Geschwulst ansehen. Sonst kennen wir doch als Charakteristikum der wahren Geschwulst ein wenigstens bis zum gewissen Grade ausgeprägtes atypisches Wachstum, wie z. B. beim gewöhnlichen Angiom, bei dessen Komponenten wir kaum je einen annähernd normalen Gefäßwandbau erkennen können. Aber noch eine weitere Tatsache spricht gegen die Annahme einer wahren Geschwulst für viele Fälle. Es handelt sich dabei um eine klinisch-therapeutische Erfahrung. Nicht ganz selten wurde nämlich beobachtet, daß sich nach teilwe scr Exstirpation einer solchen Neubildung die zurückgebliebenen Gefäßkonvolute zurückbildeten und Schlängelung und Dilatation der Gefäße zurückging. Auf diese merkwürdige Tatsache haben schon früher $\mathrm{K}$ örte und $\mathrm{Clairmont}$ (5) aufmerksam gemacht und hat Fromme (13) neuerdings wieder auf dieses eigenartige Verhalten mancher sogenannten Rankenangiome hingewiesen. Beobachtet man sonst jemals, auch bei gutartigen Tumoren, cine Rückbildung eines im Körper zurückgebliebenen Teiles der Geschwulst? Doch wohl kaum. Und die Tatsache, daß es sich oft nicht um eine umschriebene Tumorbildung, sondern ein Eingriffensein eines ganzen Arterienbaumes, ja um ein ganzes Arteriengebiet handelt, spricht meines Erachtens in nicht geringem Grade gleichfalls gegen die Annahme einer wahren Geschwulstbildung.

Ist man trotz allem geneigt, die geschlängelten Arterienkonvolute als Neubildung anzusprechen, so könnte dieses nur aus oben dargelegten Grïnden im Rahmen des Begriffs eines Hamartoms, einer Fehlbildung ođer überzähligen Anlage eines Arterienbaumes, nicht einer wahren Geschwulst geschehen. Ich möchte betonen, daB auch Aschoff (r) die in Frage stehenden Gefäßbildungen in diesem Sinne auffassen möchte.

Einige Untersucher haben die so häufig beobachtete Schlängelung und Dilatation in der näheren oder weiteren Umgebung 
der „Hauptgeschwulst“ oder der zuführenden Hauptstämme der Arterien und kleinen aneurysmatischen Bildungen als sekundäre Veränderungen, als eine Folge der Geschwulst angesprochen und auf verschiedene Weise $z u$ deuten versucht. $Z u$ den sekundären Veränderungen wurden auch Dilatation und Hypertrophie sowie Schlängelung der zugehörigen Venen gerechnet, so daß nach Verschwinden des zwischen Arterien und Venen liegenden Kapillargebietes immer breitere und ausgedehntere Kommunikationen zwischen Arterien und Venen entstehen sollen, und so angeblich ein Zustand bewerkstelligt wird, der dem Aneurysma arteriovenosum annähernd gleich kommt. Wagner läßt die Frage offen, $o b$ es sich bei all den sogenannten sekundären Veränderungen „um rein mechanische Zirkulationsfolgen handelt oder ein Übergreifen der Geschwulstbildung auf die zu- und abführenden Venen.

Die letzte Erklärung scheint mir sehr gesucht; denn man kann sich kaum vorstellen, wie eine arterielle echte Geschwulstbildung auf die Venen in dem gedachten Sinne übergreifen und sich das Fortschreiten der Geschwulst immer nur an die vorgezeichneten Bahnen der Venen halten soll. Viel eher kann man sich als Ursache der Veränderungen mechanische Momente denken, z. B. im Sinne des arterio-venösen Aneurysmas, indem sich, vielleicht nicht gerade auf dem von Wagner gedachten Wege des Kapillargebietes, aber doch an anderen Stellen Verbindungen zwischen Arterien und Venen herstellen und das Blut aus den Arterien in diese hineingepumpt wird, die Venen durch die funktionelle Inanspruchnahme zur Hypertrophie, Dilatation und Schlängelung geführt werden, und daß auf diese Weise der Eindruck erweckt wird, als ob es sich tatsächlich um massenhaft neugebildete Arterienäste handelt.

$\mathrm{Daß}$ auf ,diesem Wege, d. h. auf dem des arteriovenösen Aneurysmas, tatsächlich nicht nur ,klinisch-sekundär", sondern primär Bilder entstehen können, die auch anatomisch zunächst mit einem Rankenangiom verwechselt werden können, hat erst kürzlich Fromme (1.c.) gezeigt. Er. hat eine dem arteriellen Rankenangiom täuschend ähnliche Gefäßerkrankung am Arm beobachtet, die durch ein traumatisch entstandenes arterio-venöses Aneurysma der A. und V. subclavia mit zentralem Verschluß der 
Vene entstanden war. Dadurch hatten sich am Arm zahlreiche geschlängelte und pulsierende Stränge ausgebildet, die an einer Stelle eine Drucknekrose der Haut mit arterieller Blutung erzeugten, - Symptome, die charakteristisch sind für das Rankenangiom. $\mathrm{Daß}$ es sich aber größtenteils nicht um pulsierende Arterien, sondern um unter dem Einfluß funktioneller Inanspruchnahme veränderte Venen gehandelt hat, zeigte die Operation, bei der sich die Öffnung zwischen Arterie und Vene fand, durch die das Blut immer wieder in die Venenverzweigungen hineingepreßt worden war.

Es liegt meines Erachtens nun durchaus nahe, anzunehmen, daß auch in anderen Fällen sogenannter arterieller Rankenangiome die Diagnose einer Neubildung von Arterien auf derartig veränderte Venen zurückzuführen ist. Das würde insbesondere für die Fälle zutreffen, bei denen als Ursache der Neubildung ein Trauma angenommen wurde, die Gefäßerkrankung einen mehr diffusen Charakter hat und bei denen auch schon andere Autoren eine große Ähnlichkeit mit dem arterio-venösen Aneurysma hervorgehoben haben.

Wenn ich das Gesagte kurz zusammenfassen darf, so ergibt sich meines Erachtens Folgendes: Nicht alle Gefäßerkrankungen, die unter dem Namen des arteriellen Rankenangioms beschrieben sind, sind als solche, d. h. als wahre Neubildungen anzusehen. Es mag Fälle geben, in denen es sich zweifellos um eine Neubildung von Arterien handelt; doch sind diese nur im Sinne einer Fehlbildung oder überzähligen Anlage zu deuten, sie sind kongenital angelegt. Daß es aber ganz gleichartige Bildungen gibt, die z. B. während des Lebens auf dem Boden eines arteriovenösen Aneurysmas erworben und entstanden sind, beweist die Beobachtung von Fromme. Man darf aber nicht verlangen, daß solche traumatisch oder sonstwie entstandene arteriovenösen Aneurysmen so augenfällig sind, wie im Fall Fromme Denn es ist durchaus denkbar, daß Verbindungen zwischen Arterien und Venen auch an kleineren Gefäßen zustandekommen können, wie es ja ebenso bekannt ist, daß sich Gefäße, ähnlich wie die V. subclavia im Fall Frommes durch Unterbindung, auch spontan durch Thrombosierung verschließen können und so 
der gleiche Mechanismus entstehen würde, wie in der geschilderten Beobachtung von Fromme.

Und schließlich möchte ich glauben, daß es nicht einmal dieses Mechanismus zum Zustandekommen des in Frage stehenden Krankheitsbildes bedarf. Vielmehr möchte ich annehmen, daß es zu ähnlichen Bildungen kommen kann, wenn man annimmt, daß es sich primär um eine vielleicht konstitutionell oder toxisch bedingte Schädigung der nervösen Elemente der Gefäßwände und erst sekundär durch mechanische Momente beeinflußte Schwäche in einem oder anderm Arteriengebiet handelt; man ver. gleiche die Versuche von Lapinsky ${ }^{1}$ ) und Lewaschew ${ }^{2}$ ), die experimentell durch Schädigung der Vatomotoren ähnliche Gefäßveränderungen bekamen, wie in meiner und anderen Beobachtungen. Es wäre dann auch leicht erklärlich, daß es zunächst zu einer Hypertrophie der Gefäßwand, dann zu Dilatation und Schlängelung der Arterien eines bestimmten Verzweigungsgebietes und zur Bildung kleiner und kleinster aneurysmatischer Erweiterungen kommt die dann sekundär bersten und Blutungen verursachen können.

Es mag sich dabei um Veränderungen der Arterienwand handeln, wie sie bei der einen oder anderen Form der Arteriosklerose auftreten, die ja auch durchaus nicht immer das ganze arterielle Gefäßsystem, sondern of t nur einzelne Gebiete derselben befällt. So brauchte man für viele Fälle keine Erklärung für „sekundäre" Veränderungen beim Rankenangiom; vielmehr ließen sich alle Gefäßveränderungen auf einheitlicher Basis erklären.

Was letzten Endes die Ursache für die Gefäßwandschwäche sein mag; wissen wir heute noch nicht. Aber ich glaube doch, daß man dereinst vielleicht ebenso wie für manche unklare Erscheinung in der Ätiologie der Arteriosklerose auch hier wird auf experimentellem Wege Klärung schaffen können.

Daß Bildungen vom Charakter des Rankenangioms auch auf

1) Lapinsky, Zur Frage über die Betciligung der Nervenstämme der hint. Extremität an der vasomot. Innervation der distalen Gebiete derselben. Virch. Arch. I906, Bd. 183. - Zur Frage von der Degeneration der Gefäße bei Läsion des N. sympathicus. Deutsche Zeitschr. f. Nervenheilk. $1900, \mathrm{Bd} .16$.

2) Lewaschew, Experimentelle Untersuchungen über die Bedeutung des Nervensystems bei Gefäßerkrankungen. Virch. Arch. 1883, Bd. 92. 
dem Boden einer entzündlichen Arterienwandveränderung, einer infektiösen Arteriitis entstehen können, zeigte eine Beobachtung von Reverdin (23), der ein Aneurysma cirsoides der Augenbrauengegend im Anschluß an eine gastroenteritische Infektion be. schrieben hat. Er nimmt an, daß von der infektiösen Arteriitis ein Thrombus zurückgeblieben sei, der als Ursache des Aneurysmas aufgefaßt werden müsse. Er glaubt auch für andere Fälle des Rankenangioms bzw. Rankenaneurysmas eine solche entzündliche Ursache annehmen zu müssen.

Was nun schließlich den von mir beobachteten Fall anbetrifft, so möchte ich darüber nur noch Folgendes sagen: Die scheinbare Vermehrung der Arterienäste im Verzweigungsgebiet $\operatorname{der} \mathrm{A}$. profunda femoris und obturatoria erfuhr bei genauerer Betrachtung keine Bestätigung. Alle histologisch untersuchten Gefäße entsprachen im wesentlichen dem normalen Arterienbild. Andererseits zeigten die Arterienwände teilweise Hypertrophie (Intimawucherungen und Verdickungen der Media), teilweise degenerative Prozesse (Bindegewebsvermehrung in der Media, Narben in den Gefäßwänden, kleine Degenerationsherde); diese Narben in den Gefäßwänden sprechen ebenso wie die Pigmentablagerung im umgebenden Gewebe dafür, daß auch schon früher kleinere Gefäße geplatzt sind und zu Blutungen geführt haben. SchlieBlich fanden sich teilweise oder vollkommene thrombotische Verschlüsse des Arterienlumens. Weiter sah man an mehreren Stellen kleine sackartige Aneurysmen, von denen eines zum Platzen gekommen war und die große Blutung verursacht hatte. Ein arterio-venöses Aneurysma hat sich nicht nachweisen lassen, was aber nicht gegen das Bestehen eines solchen an einer oder anderen der kleinen Arterien spricht, insbesondere, weil man ja bei einer Operation nicht eine so gründliche Analyse aller Veränderungen vornehmen kann, wie an einem anatomischen Präparat. Der histologische Befund an den Arterien spricht dafür, daß es sich bei dem durch lange Krankheit sehr geschwächten Mann um primär-degenerative Prozesse im beschriebenen Arteriengebiet gehandelt hat, eine Gefäßwandschwäche, die im Verein mit mechanischen Momenten. die durch die Thrombenbildung in einzelnen Gefäßen beeinflußt sein mag, zu Veränderungen geführt hat, die dem Angioma arteriale racemosum sehr ähnlich oder gleich sind. 
Wenn ich mir auch bewußt bin, daß jeder Beobachter am stärksten unter dem Eindruck der persönlich gesehenen und untersuchten Beobachtung steht, so glaube ich doch, auf Grund einer möglichst objektiven Betrachtung anderer Veröffentlichungen ähnlicher Art, daß die Entstehung einer ganzen Reihe einschlägiger Fälle an den verschiedensten Körperstellen auf ähnliche Veränderungen der Gefäßwände zurückgeführt werden könnten und so die Zahl der Fälle, die als Neubildungen auf Grund überzähliger Gefäßanlagen anzusprechen wären, immer geringer würden. Und sicherlich ist manche Diagnose älterer Zeit auf Neubildung von Arterien im Bereich eines Rankenangioms nur deswegen fälschlicherweise gestellt worden, weil man nicht wußte, daß Venen sich unter funktioneller Inanspruchnahme zu arterienähnlichen Gefäßen umwandeln können, wie es uns durch die Untersuchungen von $F$ is cher und $S \mathrm{ch}$ mieden (I I) und $S$ tich und $Z$ oeppritz (30) gelehrt worden ist. Nur unter diesem Gesichtspunkt ist die fehlerhafte Anschauung Wagners zu erklären, der von einem Übergreifen der arteriellen Geschwulst auf die Venen sprach.

\section{Literaturverzeichnis.}

I) A s c h off, Geschwülste. I909, Bd. I.

2. Baum, De laesionibus aneurysmatibusque arteriarum glutaeae et ischiadicae. Inaug.-Diss. Breslau 1859.

3. Borchardt, Aneurysma cirsoides am linken Arm. Münchner med. Wochenschr. 1904, S. 1078.

4. Breschet, Ein Fall von Ang. art. racem. am Unterschenkel und Fuß. Mém. de l'Acad. de méd., T. 3, S. 183.

5. Cla irm ont, Zur Behandlung des Ang. art. racem. Arch. f. klin. Chir., Bd. 85, S. 589 .

6. De et $z$, Ang. art. racem. im Bereich der Art. corpor. callosi. Virchows Arch., Bd. 168, S. 34 I.

7. Drysda le. Lancet 1904, 9. I. S. 96 , siehe auch Simmonds, Virchows Arch., Bd. 180 .

8. Edmunds. Lancet $1884, I 1,776$.

9. Em a $\mathrm{n}$ u e 1, Ein Fall von Ang. art. racemos. des Gehirns. Deutsche Zeitschr. f. Nervenheilk., Bd. 14.

I0. Engelbrecht, Ang. art. racemos. Arch. f. klin. Chir., Bd. 55 .

II. Fis cher u. Schmieden, Experim. Untersuchung. über die funkt. Anpassung der Gefäßwand usw. Frankfurter Zeit. f. Path. 1909, Bd. 3.

12. Froehlich, Ein Fall von Rankenang. an d. unt. Extrem. Inaug.Diss. Breslau 1902. 
13. Fromme, Über die Beziehungen des Aneurysma art. venos. zum Ang. art. racemos. Bruns' Beitr. 1919, Bd. I14, S. 57.

14. Gabryszewski, Aneurysma cirsoid. d. Armes u. Vorderarmes durch zahlreiche Unterbindung und Kauterisation geheilt. Lwowski typ. lek. 1913, Nr. 13. Zentralbl. f. Chir. 1914, 20.

15. H e ine, Über Ang. art. racemos. am Kopf und dessen Behandlung. Prager Vierteljahrszeitschr., Bd. I03 u. I04.

I6. Is ra e l, Angiektasie im Stromgebiet der Art. tibial. ant. Arch. f. klin. Chir. 1880, Bd. 13, S. 24.

17. Ka lis che r, Demonstr. des Gehirns eines Kindes mit Telangiektasien der linksseit. Gesichtskopfhaut u. Hirnoberfläche. Berliner klin. Wochenschr. I897, S. 1059 .

18. Keppler, Zur Behandlung des Ang. art. racemos. Bruns' Beitr., Bd. $78, \mathrm{H} .3$.

19. Körte, Beiträge zur Lehre vom Ang. art. racemos. Deutsche Zeitschr. f. Chir., Bd. I3, S. 24.

20. - Oper. des Ang. art. racemos. capitis. Zentralbl. f. Chir. I907, Nr. 23, S. 664 .

21. Nicoladoni, Phlebarteriectasia spontanea pedis. Arch. f. klin. Chir. 1875 , Bd. 18. S. 7 I1.

22. $\mathrm{P}$ a $\mathrm{n}$ as, Ein Fall von Ang. art. racemos. an der Hinterbacke. Gazette des hôpitaux 1872, 91 .

23. Reverdin, Anevrysme cirsoide d'origine infectieuse. Revue de Chir. Paris I897. Revue méd. de la Suisse Rom. Genève 1898. Zentralbl. f. Chir. 1898 .

24. S chm it t, Beitr. z. Klinik u. chir. Behandlung des Ang. art. racemos., besonders des Kopfes. Bruns' Beitr., Bd. 118, S. 178 .

25. S c hü ck, Über Wesen und Entstehung des Ang. art. racemos. Inaug.Diss. Berlin 1885 .

26. Si e bold, v., Ein Ang. art. racemos, am FuB. Sammlung seltener und auserlesener chirurgischer Beobachtungen und Erfahrungen. Rudolstadt 1807 , Bd. 2, S. 341 .

27. Si egmund, Zur Behandlung des Ang. art. racemos., besonders des Kopfes. Deutsche Zeitschr. f. Chir. 1893, Bd. 37, S. 236.

28. S i m monds, Über das Ang. racemosum und serpentinum des Gehirns. Virchows Arch., Bd. 180.

29. Ste in heil, Über einen Fall von Varix aneurysmaticus im Bereich der Hirngefäße. Inaug.-Diss. Würzburg 1895.

3o. Stich u. Zoeppritz. Beitr. z. path. Anatomie u. allg. Pathol. I909, Bd. 46.

31. Virch ow, Die krankhaften Geschwülste. I867, Bd. 3.

32. W a g n e r, Über das art. Rankenang. an der ob. Extrem. Beitr. z. klin. Chir. I 894, Bd. I I, S. 49.

33. Wilms, Ein Fall von Ang. art. racemos. der Kniescheibe. Meyers „Elektrizität und ihre Anwendung auf die praktische Medizin“. 